Cipango Cahiers d'études japonaises

$17 \mid 2010$
La péninsule retrouvée

\title{
Conversation au fil du pinceau entre lettrés de l'archipel et de la péninsule
}

Arai Hakuseki et les envoyés coréens à Edo en 1711

Brush Talk between Literati from Both Sides of the Sea: Arai Hakuseki and

Korean Envoys to Edo in 1711

\section{Vincent Grépinet}

\section{(2) OpenEdition}

Journals

Édition électronique

URL : https://journals.openedition.org/cipango/1120

DOI : 10.4000/cipango. 1120

ISSN : 2260-7706

Éditeur

INALCO

Édition imprimée

Date de publication : 30 juin 2010

Pagination : 63-88

ISBN : 978-2-85831-195-8

ISSN : $1164-5857$

Référence électronique

Vincent Grépinet, "Conversation au fil du pinceau entre lettrés de l'archipel et de la péninsule », Cipango [En ligne], 17 | 2010, mis en ligne le 08 septembre 2012, consulté le 30 juin 2021. URL : http:// journals.openedition.org/cipango/1120; DOI : https://doi.org/10.4000/cipango.1120

Ce document a été généré automatiquement le 30 juin 2021.

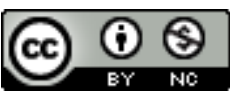

Cipango est mis à disposition selon les termes de la Licence Creative Commons Attribution - Pas d'Utilisation Commerciale 4.0 International. 


\section{Conversation au fil du pinceau entre lettrés de l'archipel et de la péninsule}

Arai Hakuseki et les envoyés coréens à Edo en 1711

Brush Talk between Literati from Both Sides of the Sea: Arai Hakuseki and

Korean Envoys to Edo in 1711

\section{Vincent Grépinet}

1 Au Japon, le regard porté sur la période d'Edo (1603-1867) a sensiblement varié depuis la fin du XIX ${ }^{e}$ siècle. À partir des années qui ont suivi la restauration de Meiji (1868), les Japonais ont eu tendance à la considérer comme une période d'obscurantisme. En revanche, entre les années 1970 et 1990, alors que le Japon était devenu une puissance financière et industrielle, on a vu une inversion des signes sous la forme d'une réinterprétation positive de cette même période, qualifiée désormais de prémoderne ${ }^{1}$. Sous le règne des Tokugawa, assimilé à une longue période de paix, le Japon aurait entretenu des relations paisibles, fondées sur la sincérité et la confiance, avec des pays voisins, en particulier avec la Corée. Mais une telle présentation donne, par son idéalisation excessive, une image faussée. À trop vouloir insister sur un âge d'or des relations, par opposition à la période sombre de la colonisation (1910-1945), on court le risque de faire l'impasse sur l'existence d'une lutte idéologique qui a pourtant longuement opposé les deux pays sur le plan diplomatique.

Certains historiens ayant longuement analysé cette opposition idéologique ${ }^{2}$, nous avons préféré en donner une illustration, à travers la traduction du Kōkan hitsudan 江關筆談, un texte attribué à Arai Hakuseki 新井白石 (1657-1725), célèbre confucianiste du début du XVIII ${ }^{e}$ siècle, qui fut également conseiller de Tokugawa Ienobu 徳川家宣 (1662-1712), sixième shōgun (il gouverne de 1709 à 1712) de la lignée Tokugawa. Ce texte, en forme de dialogue, donne un éclairage sur les débats qui opposaient les lettrés japonais aux lettrés coréens à l'époque d'Edo, et révèle, en filigrane, leur vision du monde. 


\section{Le contexte historique}

3 Depuis la période Muromachi (1333-1573), le Japon et la Corée entretenaient des relations diplomatiques, plus ou moins régulières, à travers l'échange d'ambassades ${ }^{3}$. À la fin du XVI ${ }^{e}$ siècle, Toyotomi Hideyoshi 豊臣秀吉 (1536-1598), alors maître incontesté de l'archipel nippon, mit brutalement fin à cet état de fait. Il décida d'envoyer une armée de cent cinquante mille hommes à l'assaut de la péninsule coréenne. Cette attaque marquait la première étape d'un projet plus vaste, dont on a pu dire qu'il consistait à conquérir la Chine, quoique cette thèse soit de nos jours fortement remise en question ${ }^{4}$. Les Coréens qui, jusque-là, ne tenaient pas en haute estime leurs voisins turbulents nourrirent dès lors une haine tenace contre les Japonais. Non seulement ceux-ci avaient dévasté leur territoire, mais ils avaient également emmené de nombreux prisonniers. Les Coréens venaient donc de subir une humiliation sans pareille. N'avaient-ils pas été sur le point d'être conquis par un peuple considéré comme arriéré ?

4 Après le décès de Toyotomi Hideyoshi, en 1598, Sō Yoshitoshi 宗義智 (1568-1615), seigneur du fief de Tsushima, s'empressa de reprendre contact avec la cour coréenne dans l'espoir de voir renaître rapidement les échanges commerciaux, mais sans succès ${ }^{5}$. Par la suite, avec l'aval du nouveau shōgun Tokugawa Ieyasu 徳川家康 (1543-1616), qui lui donna une sorte de blanc-seing diplomatique, il réussit à persuader la cour royale coréenne de renouer des liens avec le Japon ${ }^{6}$. Finalement, les relations reprirent, mais non sans quelques arrière-pensées des deux côtés.

5 En effet, à l'aube du XVII ${ }^{e}$ siècle, la cour coréenne était divisée en deux factions radicalement opposées. L'une refusait tout contact avec l'ancien ennemi tandis que l'autre, plus pragmatique, soulignait la nécessité de mieux connaître les agissements des barbares voisins pour parer à toute nouvelle attaque surprise, et surtout, face à la menace des Mandchous au nord, pour sécuriser la frontière sud. C'est finalement la deuxième qui devait l'emporter. Pour les Tokugawa, selon l'historien américain Ronald Toby, les relations avec la Corée entraient dans une double stratégie: sur le plan domestique, renforcer la légitimité de leur pouvoir, et sur le plan diplomatique, établir un « ordre international japonais $»^{7}$, pour s'émanciper du modèle chinois qui, jusque-là, régissait les relations entre les pays d'Asie de l'Est. Dans ce but, le bakufu fit en sorte de contrôler les points de contacts avec les pays étrangers, que les historiens japonais ont coutume d'appeler les «quatre portes $»^{8}$. Il expulsa, vers la fin des années 1630 , les Européens, sauf les Hollandais. Il mit en scène sur le territoire japonais la venue des ambassades du royaume des Ryūkyū et de la Corée afin de montrer sa capacité à faire venir des délégations étrangères, et affirmer ainsi son autorité. Toutefois, parmi les pays étrangers, seule la Corée était considérée comme un pays de même rang que le Japon, les Ryūkyū étant depuis 1609 sous le contrôle du clan des Shimazu.

6 Au début de l'époque d'Edo (1603-1867), la Corée était liée à la Chine des Ming par une relation tributaire (en coréen sadae 事大), tandis que les seigneurs de l'archipel affichaient une fière indépendance, refusant toute idée de rapport hiérarchique avec l'empereur chinois. Cette différence sensible représentait un frein sérieux aux rapports entre la Corée et le Japon. Malgré tout, Japonais et Coréens parvinrent à établir, en théorie du moins, une relation sur le principe de "voisinage » ou de "bon voisinage » (kōrin kankei 交粼関係 ou zenrin kankei 善隣関係), ce qui impliquait une idée d'égalité. Le roi de Corée envoyait une ambassade auprès de son homologue japonais, le shōgun 
Tokugawa, pour échanger des lettres officielles (shokei 書契) ${ }^{9}$. Mais en pratique, l'envoi (ou la venue) des ambassades coréennes était interprété différemment dans les deux pays. Les Coréens entendaient sonder, pour des questions de sécurité, la situation politique du pays voisin par l'entremise des ambassades. En même temps, ils s'étaient convaincus que leur pays était, depuis la chute des Ming en $1644^{10}$, le dernier bastion de la civilisation chinoise, autrement dit, une petite Chine (littéralement, shōchūka 小中 華), parce qu'on y appliquait rigoureusement le confucianisme comme principe moral et politique. À ce titre, ils se sentaient investis d'une mission civilisatrice à l'égard du pays voisin, d'où l'attention extrême portée par la cour coréenne au choix des membres des ambassades, car ces derniers devaient faire sentir la supériorité de leur culture à travers l'excellence de leur art. De leur côté, les Japonais avaient une attitude « antinomique » face à la Corée, pays officiellement égal sur le plan diplomatique mais considéré en même temps, en privé, comme inférieur au Japon ${ }^{11}$. Les membres du bakufu et les lettrés employaient fréquemment les termes raichō 来朝 (se rendre à la cour en signe de soumission) ou o-reishi 御礼使 (Ambassade de remerciement pour les bienfaits accordés $)^{12}$ pour parler de la venue d'une ambassade coréenne, laissant ainsi entendre que ses membres étaient les représentants d'un pays tributaire. Coréens et Japonais nourrissaient donc deux visions radicalement différentes de leur relation, reflets de leur ethnocentrisme respectif. Tout au long de la période d'Edo, ces divergences d'interprétation ou de perception sur la nature des relations provoquèrent des tensions diplomatiques entre les deux pays.

7 La première ambassade coréenne se rendit dans la capitale shogunale dès 1607 , suivie de onze autres, la dernière devant s'arrêter sur l'île de Tsushima en 1811. La venue de chaque ambassade était l'occasion, pour les lettrés japonais, de participer à un intense échange culturel, mais suscitait aussi la vive curiosité de la population. Il est resté de ces contacts à répétition des témoignages sous la forme d'une production littéraire et artistique considérable: notes administratives du bakufu et des fiefs, calligraphies, peintures, poèmes, récits de voyage, essais, mais aussi des «Conversations au fil du pinceau ${ }^{13}$, version on ne peut plus vivante d'un dialogue passé. Parmi ces nombreuses conversations au fil du pinceau, on trouve le Kōkan Hitsudan, fruit d'une rencontre entre Arai Hakuseki et les envoyés coréens (jap. shōtoku tsūshinshi 正徳通信使, cor. shinmyo t'ongsinsa 辛卯通信使) en 1711, à Edo, alors que ces derniers étaient venus rendre hommage au nouveau shōgun Ienobu ${ }^{14}$.

\section{Origine et nature du texte}

8 Avant cette date, Arai Hakuseki avait déjà eu l'occasion de rencontrer, à titre privé, des envoyés coréens. En 1682, il leur avait soumis un recueil de poèmes en chinois ${ }^{15}$. Les Coréens avaient alors salué sa grande maitrise de la composition et la qualité de son style. Mais à cette époque, il n'avait pas encore de fonction officielle auprès du shōgun. Il lui fallut patienter quatre ans avant de pouvoir enfin étudier auprès de son maître, l'influent Kinoshita Jun.an 木下順庵 $(1621-1698)^{16}$, qui le recommanda ensuite auprès du bakufu.

9 En 1711, son statut avait considérablement changé. Âgé de cinquante-quatre ans, il était alors « conférencier» (jikō 侍講) du shōgun. En tant que tel, il supervisa la venue de l'ambassade coréenne ${ }^{17}$ car il ressentait une vive insatisfaction concernant les relations du Japon avec la Corée. Pour Arai Hakuseki, le protocole diplomatique en 
vigueur jusque-là devait être révisé car on accordait trop d'importance à la Corée. Il avait le sentiment que les Coréens en tiraient avantage pour rabaisser les Japonais sur le plan culturel. Afin de clarifier les rapports, il décida d'y apporter des modifications avec un triple objectif : favoriser le maintien de la paix entre les deux pays, établir des relations de confiance sur un pied d'égalité, et simplifier le protocole diplomatique. Ces changements plongèrent dans un embarras extrême les envoyés coréens, mis devant le fait accompli. En effet, quelques jours seulement avant leur départ de Pusan pour Tsushima, ils apprirent qu'il fallait remplacer le titre taikun 大君 (Grand Prince) par celui de kokuō 国王 (Roi du Japon) pour désigner le shōgun dans la lettre officielle du roi de Corée $^{18}$. Par la suite, au cours de leur voyage au Japon, ils furent consternés d'apprendre que d'autres modifications avaient été apportées au cérémonial ${ }^{19}$. De ce fait, les relations entre les Japonais et les Coréens furent particulièrement tendues au cours de cette ambassade.

Ces réformes ont pu être interprétées comme un signe de mépris d'Arai Hakuseki envers la Corée et les Coréens. Pourtant, il convient de nuancer le propos à ce sujet : son attitude à l'égard des envoyés coréens semble plus complexe. Intransigeant sur le protocole, il a fait preuve, en revanche, de beaucoup de respect pour les envoyés coréens, même s'il avait conscience des divergences de point de vue, entre les deux pays, sur le plan culturel. En effet, bien avant leur arrivée à Edo, il fit parvenir un autre recueil de poèmes à Tsushima pour qu'on le soumette à leur critique ${ }^{20}$. Son intention était sans doute de montrer encore une fois, aux yeux des envoyés coréens, sa grande maitrise de la composition en chinois et donner, ainsi, la preuve du haut degré de culture atteint par le Japon ${ }^{21}$. Mais cette démarche ne reflète-t-elle pas aussi la reconnaissance implicite, par Arai Hakuseki, de l'importance de leur talent? Sinon, pourquoi prendre la peine de soumettre son travail à leur jugement? D'ailleurs, les ambassadeurs coréens, Cho T'ae-ok 趙泰億, Im Su-gan 任守幹, Yi Tong-gwak 李東郭, ne manquèrent pas de saluer unanimement son talent. En outre, si l'on considère qu'Arai Hakuseki avait peu d'estime pour les Coréens, il est alors difficile de comprendre son empressement à vouloir les rencontrer. En 1711, il s'entretint avec eux une première fois à Kawasaki, le dix-septième jour du dixième mois, puis il essaya, par la suite, à sept reprises, de parler avec les Coréens dans leur résidence à Edo, mais il ne put les voir que trois fois, entre le 17 du dixième mois et le 20 du onzième mois de la même année. À chaque fois, il leur demanda de lui offrir des calligraphies.

Peu de temps avant la rencontre qui est à l'origine du texte Kōkan hitsudan, le cinquième jour du onzième mois de 1711, les Coréens avaient remis au shōgun la lettre (kokusho 国 書) de leur roi et ils avaient assisté à la réception donnée en leur honneur le troisième jour. Ils attendaient maintenant la cérémonie de remise de la lettre de réponse du shōgun au roi de Corée. Le moment ne semblait pas propice à un dialogue, en raison de la méfiance des Coréens face aux agissements des Japonais. Pourtant, Arai Hakuseki et les dignitaires coréens entamèrent un «dialogue au pinceau ». Ce genre de pratique était fréquent entre les envoyés coréens et les lettrés japonais, c'est-à-dire entre confucianistes le plus souvent ${ }^{22}$. Chacun exprimait ses idées en chinois classique, la langue de communication internationale de l'époque en Asie orientale, une langue que tout lettré confucianiste devait maîtriser, mais qu'il ne pouvait parler. Ces dialogues n'étant pas soumis à des codes, le participant, qui d'ordinaire devait faire preuve de réserve, en raison de son rang, avait une entière liberté pour aborder tous les sujets qui lui traversaient l'esprit ${ }^{23}$. 
12 Avant de présenter en détail le contenu du dialogue, il convient de dire quelques mots sur la genèse du texte, ou plutôt des textes, car il en existe plusieurs versions. La Corée en possède deux copies très proches du Tongsa Ilgi 東嵄日記 (Récits de voyage au Japon) de Im Su-gan, l'une conservée à la Bibliothèque Nationale de Séoul, parue dans le Kugyǒk haehaeng ch'ongjae 国譯海行㧾載 (Édition intégrale des récits de voyage en coréen moderne $)^{24}$, et l'autre au Kyujanggak 奎章閣, l'ancienne bibliothèque royale. La version japonaise est, quant à elle, mentionnée dans le Catalogue général des ouvrages nationaux (publiés avant 1867) ${ }^{25}$. On y apprend ainsi que le texte, attribué à Arai Hakuseki et Cho T'ae'ok, a été recopié au début de l'ère Kansei pour être ensuite inclus dans les œuvres complètes de Arai Hakuseki ${ }^{26}$. Ce texte est connu au Japon sous le nom de version $k a n s e^{27}$ ou encore version Cho T'e-ok, du nom de l'ambassadeur coréen qui serait à l'origine du texte, par opposition au texte coréen de Im Su-gan, désigné sous le nom de version tōsa (prononciation japonaise de tongsa).

Ces deux versions, tōsa et kansei, présentent des différences notables tant par la forme que par le contenu, comme l'a parfaitement analysé Yi Il-jae. Après avoir effectué une comparaison minutieuse des deux textes et compté le nombre de caractères, il en tire la conclusion qu'on peut presque parler de deux textes distincts ${ }^{28}$. Il n'est pas question ici de présenter toutes les différences, mais simplement de donner quelques exemples pour montrer les incertitudes qui planent sur l'origine des textes.

Il existe des passages que l'on trouve uniquement dans la version tōsa. Dans la préface, absente de la version japonaise, on désigne le shōgun sous le titre de ilbon kugwang 日本 國王, «Roi du Japon ». Or dans les autres récits de voyage des envoyés coréens, il est toujours désigné par celui de kanpaku 関白, «Régent ». Dans cette même préface, on a des informations sur le lien très proche qui unit le shōgun à Arai Hakuseki. Ce dernier est mentionné sous son titre entier (contrairement à la version japonaise), ce qui est une marque de respect pour le personnage.

Dans la version kansei, plus courte, n'apparaissent pas les nombreuses tournures spirituelles qui ont été échangées entre les participants, ni les propos élogieux émis par les Coréens à l'encontre d'Amenomori Hōshū 雨森芳洲 (1668-1755), confucianiste en charge des affaires diplomatiques du fief de Tsushima et ancien condisciple d'Arai Hakuseki, lorsque celui-ci étudiait sous la direction de Kinoshita Jun.an. De plus, certains passages ont été modifiés. Ainsi, alors que dans la version tōsa, Hakuseki propose aux envoyés coréens de leur offrir une mappemonde, dans la version kansei, il leur propose seulement de leur montrer la carte.

Comment expliquer toutes ces divergences? L'absence d'information sur la genèse des textes, version tōsa ou kansei, ne nous permet pas d'avancer des affirmations au sujet de leur rédaction. Tout au plus, peut-on émettre des suppositions comme élément de réponse. Yi Il-jae avance ainsi quatre possibilités :

1. Si l'on se réfère à la préface de la version tôsa, sur le chemin de retour vers la Corée, alors que les envoyés étaient bloqués à cause d'une tempête à Shimonoseki, Im Su-gan, par désœuvrement, aurait remis de l'ordre dans les feuillets, rassemblé le tout et donné à l'ensemble le titre de Kōkan hitsudan. Il aurait envoyé le texte à Arai Hakuseki qui a pu ensuite le remanier pour donner la version kansei;

2. Il est possible qu'Im Su-gan ait recopié le texte qu'il a envoyé à Hakuseki. Im Su-gan a pu remanier par la suite le texte original en ajoutant, par exemple, une préface;

3. Il se peut également qu'Im Su-gan et Arai Hakuseki aient remanié tous les deux le texte original ; 
4. Enfin, Im Su-gan a pu envoyer le texte à Arai Hakuseki qui l'a modifié à son tour pour le renvoyer à Cho t'ae-ok. Ce dernier a pu apporter une touche finale, d'où l'attribution du texte à $\mathrm{Cho}^{29}$.

19 Quel que soit le processus de rédaction des textes, il est impossible de déterminer avec certitude quel est, des deux, l'original. Il est même probable qu'ils représentent deux versions remaniées d'un même manuscrit disparu. Seule certitude à la lecture des deux textes, les Coréens et Arai Hakuseki ont cherché, par des ajouts, des suppressions et des modifications, à mettre en avant la culture de leur pays respectif.

En effet, lorsqu'on regarde la version kansei, il ne fait pas de doute que Arai Hakuseki est lui-même à l'origine de certains passages supprimés. Ses motivations pouvaient être de plusieurs ordres: sachant que ce texte serait lu par les générations futures, il souhaitait probablement laisser une image positive de lui-même en s'attribuant un rôle central. Il a pris des précautions pour que les passages relatifs à son amitié avec le shōgun n'apparaissent pas dans cette version, soit parce qu'il ne trouvait pas utile de faire connaître ces informations ou plus probablement parce qu'il redoutait la jalousie des autres membres du bakufu en affichant cette amitié dans un texte ${ }^{30}$. Il ne voulait pas non plus citer le long passage relatif à son condisciple, Amenomori Hōshū, envers lequel il gardait une certaine rancœur, à cause de l'opposition farouche de ce dernier aux réformes protocolaires que Hakuseki avait proposées avant la venue des représentants coréens ${ }^{31}$. Plus tard, dans sa célèbre autobiographie, il devait même traiter Amenomori Hōshū de "confucianiste mal dégrossi $»^{32}$. De plus, soupçonnant les Coréens de vouloir montrer leur supériorité par la maîtrise des classiques chinois, il ne désirait pas leur laisser, même dans un texte, le loisir de faire étalage de leurs connaissances.

21 Enfin, on peut émettre une dernière hypothèse au sujet de la version kansei. Puisque cette version date de la fin du xvIII ${ }^{e}$ siècle, il est aussi possible que celui qui est à l'origine de la copie ait à son tour effectué une sélection des passages qui lui semblaient les plus intéressants.

En résumé, avant d'entreprendre la lecture du Kōkan Hitsudan, il convient de garder à l'esprit qu'il y a plusieurs versions du texte, qu'elles présentent des divergences notables et que leurs auteurs ont cherché à se présenter sous un jour favorable.

\section{L'intérêt du texte}

23 Le Kōkan hitsudan n'est pas, de prime abord, un texte facile d'accès, bien qu'il ne fasse en tout que quelques pages. Non seulement il est écrit en chinois classique, mais il faut aussi deviner qui se cache derrière les noms qui y figurent, chacun utilisant un nom de plume. En outre, comme il est de coutume entre confucianistes, il est très souvent fait référence à des ouvrages classiques chinois. La translittération des noms propres ou des titres d'ouvrage, en japonais, en coréen ou en chinois s'en trouve compliquée. On peut toutefois savourer les qualités intrinsèques du texte, au-delà des difficultés de traduction. Jusqu'à présent, les chercheurs japonais ou coréens se sont souvent contentés d'en citer quelques passages pour évoquer l'ethnocentrisme ou le protonationalisme des Coréens, ou des Japonais, à l'époque d'Edo ${ }^{33}$. Seul Miyasaki Michio a donné une analyse plus approfondie de la version kansei ${ }^{34}$. Yi Il-Jae a souligné, quant à lui, la nécessité d'étudier de front les deux textes pour éviter de tirer des 
conclusions hâtives. Dans ce but, il va même jusqu'à présenter une troisième version du texte obtenue à partir des deux textes japonais et coréens ${ }^{35}$. Cependant, un tel travail nous semble complexe. Ne vaut-il pas mieux regarder de près chaque texte séparément pour en faire ensuite une comparaison?

Nous avons donc préféré nous limiter à la présentation de la version kansei, du volume quatre des œuvres complètes d'Arai Hakuseki, tout en nous référant parfois à la version tōsa. Le Kōkan hitsudan mérite d'être étudié non pas partiellement, comme on l'a fait jusqu'à présent, mais dans sa totalité, car il a une fraîcheur que l'on trouve rarement dans d'autres écrits de l'époque d'Edo, en raison même de sa forme dialoguée. Surtout, il révèle un autre aspect des relations entre Coréens et Japonais en 1711 : malgré les tensions diplomatiques, il y a aussi eu des échanges fructueux, presque amicaux, entre $\mathrm{eux}^{36}$.

Le Kōkan histudan peut donc être interprété comme une confrontation, intellectuelle et personnelle, entre plusieurs individus conscients de leur rang et de leur valeur, et dont on devine la psychologie à travers leur propos ; il peut également être envisagé comme l'expression d'un ethnocentrisme, d'un protonationalisme, naissant à travers une confrontation culturelle. De plus, il nous donne un éclairage important, au niveau historique voire géopolitique, sur l'état et la nature des relations entre le Japon, la Corée mais aussi la Chine. Les thèmes abordés au cours du dialogue par les envoyés coréens et Arai Hakuseki sont les suivants ${ }^{37}$ :

1. L'existence au Japon d'un texte chinois ancien ;

2. Les connaissances en géographie de l'époque ;

3. Les mœurs en Asie orientale ;

4. Le style vestimentaire ;

5. Les informations personnelles relatives aux envoyés coréens.

\section{L'existence d'un texte chinois ancien}

Le dialogue s'ouvre sur l'existence ou non au Japon d'un ouvrage classique chinois, le Shang shu 尚書 ou Classique de l'Histoire, qui, selon une légende, aurait été apporté dans l'archipel, par un lettré chinois, Xu Fu 徐福, sous la dynastie Qin (221 à 207 avant notre ère). Interrogé sur cette question, Arai Hakuseki répond par l'affirmative, mais il prétend aussitôt qu'il ne peut pas le leur montrer pour des raisons, à l'évidence, peu convaincantes aux yeux des envoyés. Devant l'insistance de ces derniers, il se met à faire un long développement sur les tribulations du texte classique chinois, pour finalement conclure qu'il n'est pas nécessaire de voir une version ancienne d'un texte classique si l'on en connaît une version moderne. Il est curieux de voir un tel exposé car, à n'en pas douter, Hakuseki devait bien savoir que les envoyés, bercés dès leur plus tendre enfance par une culture confucéenne, devaient sans aucun doute connaître cette histoire. Par ailleurs, en dépit de son exposé, les envoyés restent sceptiques, puisque Hakuseki n'a pas clairement répondu à leur question. Ajoutons encore que dans la version tōsa, il n'y a nulle trace de cet exposés ${ }^{38}$. Faut-il y voir le désir de Hakuseki de faire étalage de ses connaissances, afin de montrer ainsi que les Japonais n'ont rien à envier aux Coréens dans le domaine de la culture? La suite du dialogue tend à le prouver. 


\section{Sur les connaissances en géographie de l'époque}

Arai Hakuseki enchaîne brusquement son propos sur les pays étrangers, comme pour éblouir ses interlocuteurs, par sa connaissance du vaste monde.

Ayant reçu l'ordre officiel de vous rendre [au Japon], vous avez franchi la mer pour venir jusque [chez nous] et vous avez surmonté beaucoup de difficultés pour le bien de nos deux pays. N'est-ce pas formidable? Quant à moi, [j'ai l'impression] d'avoir passé toute ma jeunesse au fond d'un puits car je n'ai jamais eu l'occasion de voir la mer, mais [ensuite] devenu adulte, j'ai rencontré deux ou trois représentants de votre pays en 1682. Plus tard, j'ai rencontré des Chinois, [des représentants] des Ryūkyū, des habitants de pays de l'Atlantique, d'Europe, tels que des Hollandais, des Suédois ou des Italiens qui sont venus [chez nous]. Et maintenant, je passe la journée à m'entretenir avec vous. C'est comme si j'avais reçu le privilège de faire le tour du monde.

Si Hakuseki félicite les envoyés pour leur long voyage, c'est pour mieux faire valoir que lui n'a pas eu besoin de se déplacer pour rencontrer des étrangers. On peut y voir l'intention, de manière détournée, de placer le Japon sous un jour favorable, au centre du monde, car les représentants étrangers se déplacent vers lui, tandis que la Corée reste tributaire de la Chine et envoie des ambassades au Japon. Comme Hakuseki le fait remarquer, ce n'est pas sa première rencontre avec des envoyés coréens. Il évoque celle de 1682, dont on trouve un récit dans son ouvrage autobiographique :

À cette époque, je fis la connaissance d'un confucianiste du nom d'Abiru du fief de Tsushima. À vingt-six ans, au printemps, j'occupai de nouveau une fonction, et cette année-là en automne, une ambassade coréenne vint [au Japon]. Grâce à Abiru, je rassemblai une centaine de poèmes dans un recueil que je soumis au jugement des trois grands envoyés. Ils déclarèrent vouloir me rencontrer et écrire ensuite une préface. Le premier jour $d u$ neuvième mois, je me rendis dans leur lieu de résidence et je rencontrai l'attaché Sŏngwan, le rédacteur Yi Tam-nyong et l'officier Hong Se-t'e. Nous écrivîmes des poèmes et ce soir là, Sŏngwan me remit mon recueil de poèmes auquel il avait ajouté une préface ${ }^{39}$.

Pour Hakuseki, qui était d'origine modeste, cette rencontre marqua une étape importante dans sa carrière. En effet, dans la société japonaise où le néoconfucianisme de Zhu Xi occupait une grande place ${ }^{40}$, le jugement ou les écrits d'un lettré coréen étaient respectés et admirés par la plupart des confucianistes, car la Corée était considérée comme le deuxième berceau du confucianisme, après la Chine, depuis que Fujiwara Seika 藤原惺窩 (1561-1619) avait contribué au développement du néoconfucianisme au Japon. Pour un lettré japonais ambitieux, la préface écrite par un pair coréen servait de passeport pour une grande carrière, d'où l'empressement des confucianistes japonais, tout au long de la période d'Edo, à vouloir aller à la rencontre des ambassades coréennes. Cela se vérifie avec Hakuseki, dont la renommée remonta en effet jusqu'aux oreilles de l'influent Kinoshita Jun.an, qui décida de le prendre sous sa protection. Moins de trente ans plus tard, l'attitude de Hakuseki envers les Coréens aura changé, sans doute parce qu'il voulait renforcer l'autorité du gouvernement shōgunal ainsi que le prestige du Japon face à ses voisins. Néanmoins, il semble avoir gardé une certaine affection pour les Coréens qu'il avait rencontrés jadis, tel que Sŏngwan, l'auteur de la préface de son premier recueil de poèmes, car il demande de ses nouvelles à la fin du dialogue.

Après avoir rappelé sa rencontre avec les envoyés coréens, Hakuseki donne une liste de noms de pays étrangers, dont l'évocation laisse dubitatifs ses interlocuteurs, qui avouent leur complète ignorance à leur sujet. Si Hakuseki a une telle connaissance sur les pays éloignés, c'est qu'il a eu l'occasion, deux années plus tôt, en 1709, d'interroger 
le jésuite Giovanni Battista Sidotti (1668-1715) qui avait débarqué clandestinement sur le territoire japonais. Après cette rencontre, Hakuseki avait mis par écrit, pour un usage personnel, toutes les informations qu'il avait apprises du jésuite sur les pays occidentaux ${ }^{41}$. C'est encore l'occasion pour Hakuseki de montrer qu'il a une meilleure connaissance du monde que les Coréens, car il leur demande, non sans malice: « N'avez-vous pas de mappemonde dans votre pays?» Et les Coréens de répondre par la négative. Il cite encore des ouvrages chinois qui ne sont pas arrivés jusqu'en Corée. Curieusement, alors que le Japon a toujours gardé une relation distendue avec la Chine pour finalement rompre tout contact officiel à partir de la fin du xVI e siècle, il semble mieux fourni en textes chinois que la Corée, alors que celle-ci n'a pratiquement jamais cessé d'envoyer des ambassades en Chine. On voit donc bien que le gouvernement shogunal, contrairement à la Corée ${ }^{42}$, n'a jamais eu l'intention de se couper totalement de l'extérieur et qu'il souhaitait rester au courant des affaires du monde, en faisant remonter les informations sur les pays étrangers via Nagasaki notamment. On reproduisit même « des mappemondes en format réduit d'une grande précision ", selon Hakuseki. Les Coréens continuent la conversation en interrogeant Hakuseki sur la distance qui sépare le Japon des Ryūkyū, ou Nagasaki de la ville chinoise de Fujian, sur la piraterie ou encore sur le missionnaire italien Matteo Ricci (1552-1610) ${ }^{43}$. Concernant le nombre de navires chinois qui se rendent à Nagasaki, Hakuseki avance un chiffre supérieur à cent soixante. Il donne ainsi une idée du trafic maritime entre la Chine et le Japon au début $\mathrm{du} \mathrm{XVIII}^{\mathrm{e}}$ siècle $^{44}$. Plus loin, la conversation glisse sur le thème des mœurs autrement dit, sur le degré de civilisation.

\section{Les mœurs en Asie orientale}

31 Interrogés par Hakuseki sur la volonté des Coréens de conserver des traditions antérieures à la période des Qing, ceux-ci répondent en substance que la Corée est le dernier bastion de la civilisation en Orient depuis la fin de la dynastie Ming en 1644. Un des envoyés déclare ainsi : "Tous les pays ont [adopté] des mœurs barbares, seul le nôtre a conservé les préceptes de la civilisation ». En effet, si le royaume coréen a été contraint de reconnaître sa relation de vassalité avec l'empire des Qing, il n'en a pas moins continué de respecter des traditions héritées de la Chine " authentique » jusqu’à la période Ming. Cet attachement aux traditions chinoises se vérifie jusque dans la tenue vestimentaire ou dans la coupe de cheveux, les Coréens arborant des habits d'un autre temps et refusant la longue natte qui caractérise les Mandchous. Il ne fait aucun doute que dans l'esprit des envoyés coréens, leur pays est le garant de la "Civilisation », et qu'à ce titre, il est placé de facto au centre du monde, sa légitimité découlant de sa capacité à conserver et à reproduire les traditions passées, perdues ailleurs. La Corée est donc à leurs yeux un modèle, à tel point que les envoyés exhortent les Japonais à suivre l'exemple de leur pays. Mais au lieu d'approuver les paroles des Coréens, Arai Hakuseki raille leurs prétentions à se considérer comme les gardiens de la civilisation chinoise. Il répond en ces termes :

[...] Mais je constate [que votre tenue] vestimentaire [date des] Ming, et ils sont loin de faire penser à ceux des Yin ${ }^{45}$. Aujourd'hui, la dynastie Qing règne en [Chine] et les choses ont bien changé. Elle applique ses règles en se conformant à ses traditions. Votre pays, tout comme le royaume des Ryūkyū, se tourne vers le nord en tant que pays tributaire. Mais vous êtes autorisés à ne pas porter des nattes ou des vêtements [à la manière des Qing]. Est-ce que cela signifie que les Qing sont d'une grande magnanimité? Ou bien, n'est-ce pas plutôt que vos deux pays puisent leur force de l'est [profitent de la présence du Japon]? 
À ses yeux, si les Coréens peuvent se targuer d'une tradition chinoise, elle remonte tout au plus à la période Ming. Autrement dit, elle n'est pas si ancienne que cela. Aujourd'hui, la Corée est tributaire des Qing, mais ces derniers leur laissent une certaine autonomie, en ne les obligeant pas à adopter leurs coutumes. Pour expliquer ce fait, Arai Hakuseki ajoute une dernière phrase sibylline. Selon l'historien Naitō Konan 内藤湖南 (1866-1934), il ferait allusion à un épisode historique qui aurait eu lieu sous le règne de l'empereur Qing, Kangxi (1654-1722). Ce dernier aurait informé les envoyés coréens venus en Chine, de son intention de mettre sur le trône de Corée un de ses fils. Les Coréens auraient alors invoqué le mécontentement des Japonais si jamais une telle chose devait se produire, car un lien particulier unissait les deux pays. Apprenant cela, l'empereur chinois aurait abandonné son projet. En évoquant cet épisode, Arai Hakuseki entend dire aux Coréens que la Corée et les Ryūkyū doivent leur salut au Japon ${ }^{46}$. Face à une telle assertion, l'absence de réaction des envoyés est plus qu'étonnante. Comment ne pas réagir en effet alors que leur interlocuteur japonais vient de réduire à néant leur argumentation? Mais les propos d'Arai Hakuseki sont-ils seulement vrais? Pour Naitō Konan, ils sont dénués de tout fondement historique car on ne trouve nulle trace dans les sources de la dynastie Qing de ce dialogue entre l'empereur chinois et les envoyés coréens. Toujours selon lui, il n'est pas impossible que Hakuseki ait repris une rumeur qui circulait à l'époque au Japon. Pourtant certains historiens japonais ne sont nullement étonnés par le silence des Coréens. Ainsi, Miyasaki Michio pense que ces derniers étaient à court d'arguments. Nullement troublé par l'absence de sources historiques à ce sujet, il avance une explication peu convaincante. Coréens et Japonais auraient gardé le silence sur cette affaire embarrassante, d'où l'absence de témoignage. Mais on trouverait, toujours selon Miyasaki Michio, une allusion à cet épisode dans une lettre du fief de Tsushima et il cite à ce propos un extrait du volume cinq des œuvres complètes de Arai Hakuseki, sans donner de référence au sujet de l'existence réelle de cette lettre ${ }^{47}$. Or dans la version tōsa, la réponse de Hakuseki, réduite à sa portion congrue, est tout aussi sibylline : «Pourquoi n'a-t-on pas d'aide du pays voisin?»

Ce passage est donc un des plus problématiques du texte Kōkan hitsudan de la version kansei, car il jette le doute sur l'ensemble du texte. On peut supposer que Hakuseki a ajouté, a posteriori, cette réponse, ou encore que les Coréens ont tout simplement supprimé un long développement gênant dans leur version. Ainsi, la présence ou l'absence de ce passage suffit à renforcer l'idée que les compilateurs des deux textes ont, chacun de leur côté, cherché à mettre en valeur la supériorité de leur pays, en ajoutant des phrases qui n'ont pas été échangées au cours de la conversation, ou en supprimant des passages délicats.

Plus loin dans le dialogue, Arai Hakuseki manifeste sa réprobation quand un envoyé lui demande maladroitement la possibilité de voir la «technique militaire » des Japonais, car il sait très bien que cette question n'est pas anodine ${ }^{48}$. En outre, cet envoyé lui a, semble-t-il, déjà fait cette requête auparavant ${ }^{49}$. Il est certain qu'à travers cette question, les envoyés coréens cherchent indubitablement à provoquer Hakuseki qui répond: "J'ai déjà entendu cette requête, et vous refaites aujourd'hui la même demande. J'ai le sentiment que vous ne considérez notre pays [simplement] comme un pays de guerriers!»

En effet, cela revient à dire pour les Coréens que le Japon ne suit pas suffisamment les préceptes confucéens car la politique y est du ressort des guerriers, alors que dans leur 
pays, le système d'examen ( $k$ wagŏ 科擧), inspiré du modèle chinois, permet en théorie à tous ceux qui en ont les capacités de faire une carrière dans la bureaucratie, où on emploie les meilleurs éléments en fonction de leur mérite. En outre, dans ce système, le concours des classiques est jugé de loin comme étant le meilleur, devant le concours militaire. En d'autres termes, le militaire est subordonné aux lettrés, d'où le mépris des envoyés coréens à l'égard de la structure sociale japonaise où l'aristocratie guerrière est prédominante.

Irrité, Hakuseki se lance alors dans une explication sur les raisons de cet état de fait dans son pays tout en faisant des parallèles avec la Chine antique, pour montrer qu'il y a des précédents, qui plus est, dans le «berceau » de la civilisation asiatique. Il souhaite démontrer ainsi que le Japon n'accorde pas seulement de l'importance à ce qui est militaire mais aussi aux lettres, que ces deux aspects ne font qu'un. Il ajoute que le Japon respecte scrupuleusement les préceptes confucéens, en particulier depuis l'instauration de la pax Tokugawa, grâce à laquelle l'archipel vit en paix depuis un peu plus d'un siècle ${ }^{50}$. Pour finir, il a soin de rappeler les dernières paroles de Sin Suk-chu 申叔舟 (1417-1475), un lettré coréen et diplomate qui s'était rendu au $\mathrm{Xv}^{\mathrm{e}}$ siècle au Japon et qui avait fait paraitre, par la suite, un ouvrage de géographie et de politique sur les pays à l'est de la péninsule coréenne. En rappelant à la mémoire des envoyés coréens les paroles de ce personnage qui avait œuvré pour le rapprochement entre les deux pays, Hakuseki tient à montrer que les Coréens ne sont pas si policés qu'il n'y paraît, puisqu'ils ont une propension fâcheuse à entretenir les dissensions par leurs allusions, et donc à faire le contraire de ce que Sin Suk-chu a dit, en insinuant des choses inacceptables pour le Japon, au lieu de travailler pour la paix. Les envoyés coréens ne peuvent alors que s'excuser. Ce qui ne les empêche pourtant pas de relancer la polémique en abordant le sujet sur les interdits touchant le nom des rois et des ancêtres. Ils reprochent aux Japonais de ne pas respecter scrupuleusement cet interdit, autrement dit, de ne pas suffisamment connaître les traditions classiques. Mais Hakuseki leur répond que ce problème vient de la langue japonaise même, et que pour l'écriture, il peut arriver qu'on emploie des sinogrammes simplement pour leur son, sans distinction du sens. Cette réponse ne rassure pas les envoyés pour autant, car ils ont des doutes sur la teneur de la lettre officielle qui leur sera remise, mais Hakuseki feint d'en ignorer le contenu. Or cette lettre fut, quelques jours plus tard, à l'origine d'un lourd différend diplomatique entre les Japonais et les Coréens ${ }^{51}$. La conversation glisse ensuite sur les tenues vestimentaires et sur les informations personnelles relatives aux membres du cortège coréen.

\section{Tenues vestimentaires et informations personnelles}

37 Là encore, les envoyés coréens tentent de montrer qu'ils sont les garants de la tradition, que leurs tenues vestimentaires sont conformes à ce qui est décrit, en particulier dans les textes de Zhu Xi. Cho T'ae-ok montre fièrement sa coiffe, un bokkin, et demande à Hakuseki s'il a déjà vu quelque chose de semblable. Hakuseki en demande un exemplaire pour en faire une copie. Dans la version tōsa, il fait la même demande mais pour pouvoir l'accrocher à son mur et «l'admirer respectueusement ». Encore une fois, on constate que les compilateurs ont choisi leur version des faits. Les Coréens font ensuite mine d'être étonnés en constatant l'absence, au Japon, de certains ouvrages, à leurs yeux majeurs, relatifs à ce sujet. Mais Hakuseki va encore une fois prendre l'ascendant sur ses pairs coréens en rappelant, à propos des rites confucéens, que le 
Japon a conservé des manières de faire qu'on ne trouve nulle part ailleurs, et il cite à ce sujet un auteur chinois du début du vIII ${ }^{\mathrm{e}}$ siècle, Lu Deming, qui mentionne ce fait. Cela revient à dire que le Japon aussi est le lieu où les traditions confucéennes sont les mieux préservées. D'ailleurs, l'auteur du texte ajoute une note qui vient étayer les propos de Hakuseki.

Ensuite, trois secrétaires se joignent au dialogue. Hakuseki leur demande alors de se présenter pour connaitre leur situation tant familiale que leur parcours "professionnel». Cette partie du texte peut sembler ennuyeuse pour le lecteur contemporain, et plus encore pour un lecteur occidental. Mais il faut garder à l'esprit qu'au début du XVIII ${ }^{e}$ siècle, en Asie du Nord-Est, ce genre d'échange entre lettrés devait être monnaie courante. Il leur permettait de se situer par rapport à leurs interlocuteurs, et c'était surtout l'occasion de glisser le nom d'un ancêtre connu, pour montrer que l'on venait d'une lignée prestigieuse, ce qui, dans une société dominée par une pensée confucéenne qui accorde une grande importance à la continuité entre les générations, était un point non négligeable. Mais on est surtout frappé de voir la réaction de Hakuseki, partagé entre l'émerveillement et la jubilation, quand il apprend que certains envoyés sont les descendants de personnages illustres qui, autrefois, sont venus au Japon. L'homme d'État cède la place à l'historien qui, constatant une continuité historique entre des acteurs qui œuvrent pour le rapprochement entre les deux pays, ne peut s'empêcher d'être fasciné par les hasards de l'histoire. Hakuseki prend également des nouvelles d'envoyés coréens qu'il a jadis rencontrés, et il se montre désolé à l'annonce du décès de l'un d'entre eux. À la fin du dialogue de la version kansei, un des envoyés coréens conclut en disant :

On peut lire dans les classiques : "Aussitôt que nous nous sommes rencontrés, nous avons ri comme de bons amis. Qui peut dire ce qui nous divise?" Au cours de la réunion d'aujourd'hui, nous avons eu l'occasion de plaisanter. C'est une chose bien rare depuis que nos deux pays entretiennent des relations. Quand les esprits s'accordent, on peut oublier complètement les divisions.

Cette allusion à la complicité entre les envoyés coréens et Arai Hakuseki ne peut être comprise à la seule lecture de la version kansei, car à aucun moment les participants ne rient ou ne plaisantent. Il faut consulter la version tōsa, pour découvrir que d'autres thèmes, sur le tabac ou l'alcool, ont été abordés au cours de leur conversation, et que des propos plus légers ont contribué à détendre l'atmosphère. Ceci met en lumière un aspect supplémentaire du processus de recomposition du dialogue. L'auteur de la version kansei aura sélectionné les passages les plus sérieux à ses yeux, et supprimé ceux qu'il jugeait futiles.

\section{En guise de conclusion}

Tout d'abord, on peut se demander pour quelles raisons Cho T'ae-ok a invité Arai Hakuseki à dialoguer directement à l'aide du pinceau. N'aurait-il pas été plus facile de recourir à des interprètes tant coréens que japonais ${ }^{52}$ ? Or, l'ambassadeur coréen avait très certainement une arrière-pensée : tester la capacité de Hakuseki à s'exprimer en chinois et vérifier sa maîtrise des classiques. Car aux yeux des lettrés coréens, il n'y a rien de plus étonnant que de voir un homme qui n'a pas passé d'examen, et qui n'a donc pas fourni la preuve de ses talents, occuper une haute fonction, comme c'est le cas au Japon. Tout au long de la période d'Edo, les envoyés n'eurent de cesse de railler le 
système héréditaire japonais selon lequel un homme peut devenir un grand seigneur ou un conseiller du gouvernement en raison même de sa naissance. Ils critiquèrent aussi les lettrés japonais pour leur maladresse dans la composition de poèmes en chinois classique, disant péjorativement qu'ils dégageaient des « relents japonais ». Ainsi, Shin Yu-han, auteur du Haeyurok, porte-t-il au milieu du XVIII ${ }^{\mathrm{e}}$ siècle, un jugement très sévère à l'égard de la famille Hayashi ${ }^{53}$ qui monopolise la fonction de chef des études (daigaku no kami 大学頭) alors qu'elle ne maîtrise qu'imparfaitement la composition chinoise ${ }^{54}$. Arai Hakuseki fait figure d'exception parmi les lettrés japonais de la période d'Edo, car il échappe aux critiques des Coréens, qui lui reconnaissent un grand talent. En 1711, l'ambassadeur coréen déclare même que ses poèmes égalent ceux des Chinois. D'ailleurs, certains de ses poèmes furent étudiés par la suite aussi bien en Corée qu'en Chine ${ }^{55}$. Arai Hakuseki maîtrise non seulement la composition en chinois classique, mais il fait preuve, comme on peut le voir dans le Kōkan hitsudan, d'une grande érudition en distillant judicieusement dans son propos des références à des classiques chinois.

41 Hakuseki devait se douter de ce qui l'attendait en se rendant dans le bâtiment réservé aux envoyés, mais sûr de lui, il a accepté sans hésiter l'invitation de Cho T'ae-ok. Pour la forme, on pourrait dire qu'il a passé « haut la main » le test de la composition ${ }^{56}$.

Sur le fond, Hakuseki a engagé « un bras de fer culturel » avec les Coréens. En effet, tout au long du dialogue, il s'efforce de prendre l'ascendant sur ses interlocuteurs, et à plusieurs reprises, il les plonge même dans l'embarras, en pointant du doigt leur ignorance sur certains sujets. Il sait pertinemment que les Coréens accordent la primauté aux lettres sur les armes. En 1716, il écrit ainsi : "les Coréens luttent au moyen des lettres et font en sorte de nous rabaisser ${ }^{57}$. Il a donc conscience qu'une lutte culturelle était engagée entre les deux pays. Au cours de la conversation avec les Coréens, il s'efforce de montrer que le Japon est certes un pays aux traditions guerrières, mais qu'on y maîtrise aussi parfaitement les lettres. En tant qu'homme d'État, il a pleinement rempli son rôle face à des envoyés d'un autre pays, en montrant que le Japon n'a rien à envier à la Corée. Cependant, il n'est absolument pas certain que tous les propos qui apparaissent dans la version kansei du Kōkan hitsudan aient été réellement échangés entre les Coréens et Arai Hakuseki, les versions kansei et tōsa présentant de grandes divergences. Certains passages ont été remaniés après la rencontre, d'autres tout simplement ajoutés. Mais si on considère que toutes les modifications de la version kansei sont de la main d'Arai Hakuseki, on peut alors émettre une hypothèse sur l'état d'esprit de l'auteur. N'aurait-il pas souhaité, quitte à recourir à une pratique déloyale en recomposant le dialogue, laisser aux générations futures une image positive de son action comme défenseur de la culture japonaise?

Enfin, quand on songe aux propos acerbes que Hakuseki tiendra par la suite sur la Corée et les Coréens dans ses textes, au point que certains historiens ont pu voir en lui un des Japonais qui a contribué à nourrir le nationalisme japonais ${ }^{58}$, on ne peut qu'être étonné du sentiment de solidarité qui se dégage des paroles de Hakuseki envers ses pairs coréens à la fin du dialogue. Pour comprendre cette attitude, a priori contradictoire, il ne faut pas perdre de vue que Hakuseki était à la fois un homme d'État, autrement dit un conseiller du prince, et un intellectuel féru de culture confucianiste. Mais il avait soin de ne pas mélanger ces deux facettes, en distinguant le général du particulier, l'érudit confucéen devant forcément céder le pas à l'homme d'État lorsque les intérêts du pays l'exigeaient. Hakuseki avait, à n'en pas douter, une 
haute estime de son rôle de conseiller, et il fit en sorte de toujours renforcer l'autorité du shōgun tant sur le plan domestique que sur le plan international. L'homme public pouvait donc avoir des mots très durs envers les Coréens, sachant que ces derniers considéraient les Japonais comme des barbares, mais le confucianiste n'oubliait pas non plus qu'il était un membre d'une communauté de lettrés avec lesquels il partageait les mêmes valeurs, ce qui explique ses propos affectueux envers les envoyés coréens sur le plan privé.

\section{NOTES}

1. Pierre-François Souyri a développé, à plusieurs reprises, ce sujet, dans le cadre d'une réflexion sur la question de la modernité japonaise. Cf. «La modernité japonaise dans tous ses états », in Le Débat, n 153, janvier-février 2009, p. 88-99.

2. Notamment Etsuko Hae-jin Kang, Diplomacy and Ideology in Japanese-Korean Relations: from the Fifteenth to the Eighteenth Century, Saint Martin's Press, New York, 1997, 312 p.

3. La lutte contre la piraterie fut une des raisons qui poussa la cour coréenne à ouvrir des relations diplomatiques avec les autorités de l'archipel, dès avant l'époque d'Edo. Les pirates connus sous le nom de Wakō 倭寇 (" envahisseurs japonais », le terme Wa désignant l'archipel ou ses habitants depuis la plus haute Antiquité. Wakō, en réalité, aussi bien en Chine qu'en Corée, désignait la piraterie en général, laquelle n'était pas uniquement "japonaise ») - trouvaient en effet refuge dans les îles de Tsushima et d'Iki, mais aussi le long des côtes occidentales de Kyūshū. Voir l'article de Guillaume Carré : http://cipango.revues.org/1118.

4. En 1592 et en 1597. En coréen, la première invasion est appelée Imjin waeran 壬辰倭乱 (Invasion Wa - japonaise - de l'année eau et dragon).

5. L'économie du fief dépendait en grande partie du commerce avec la péninsule.

6. Il n'y pas, à notre connaissance, de document prouvant clairement que Tokugawa Ieyasu ait donné ordre à Sō Yoshitoshi de faire tout son possible pour renouer des contacts diplomatiques avec la Corée. Il est plus probable, en raison de la rapidité avec laquelle les relations ont été reprises, que le clan des Sō, mu par des intérêts économiques propres, ait agi de son propre chef pour ensuite mettre Ieyasu devant le fait accompli. Nous entendons donc par «blanc-seing » le fait que le shōgun ait fermé les yeux sur les agissements des Sō, lesquels allèrent jusqu'à falsifier des documents officiels pour atteindre leurs objectifs. Voir à ce sujet Tashiro Kazui 田代和生, Kakikaerareta kokusho - Tokugawa Chōsen gaikō no butai ura 書き換えられた国書一徳川・朝鮮外交 の舞台裏, Chūkō shinsho 中公新書, Tōkyō, 1983, 220 p. Voir aussi Vincent Grépinet, Mémoire de DEA, Le fief de Tsushima et la reprise des contacts entre la Corée et le Japon 1598 - 1636, INALCO, Paris, 2004.

7. Ronald Toby, State and Diplomacy in Early Modern Japan-Asia in the Development of the Tokugawa Bakufu, Princeton University Press, Princeton, 1984, 312 p. Arano Yasunori 荒野泰典, « Nihongata Ka.i chitsujō no kenkyū »日本型華夷秩序の研究 (Recherches sur la version japonaise de l'ordre barbare-civilisé), in Asao Naohiro 朝尾直弘 et al., Nihon no Shakaishi 日本の社会史 (Histoire de la société au Japon), Vol. 1, Iwanami shoten 岩波書店, Tōkyō, 1987, 384 p.

8. Soit Matsumae, Satsuma, Nagasaki, et Tsushima. Voir Tsuruta Kei 鶴田啓, «Kinsei Nihon no yottsu no “kuchi”" 近世日本の四つの「口」(Les quatre «bouches ouvertes»du Japon à l'époque moderne), in Arano Yasunori et al., Ajia no naka no nihonshi II - gaikō to sensōアジアのな 
かの日本史II - 外交と戦争 (L'Histoire du Japon dans celle de l'Asie, vol. 2 - Les Relations entre États et les guerres), Tōkyō Daigaku Shuppansha 東京大学出版社, Tōkyō, 1992, 377 p., p. 297-314. 9. Les Coréens refusaient toutefois que les Japonais envoient une ambassade dans la capitale, de peur que ces derniers en profitent pour amasser des informations sur le pays en vue d'une nouvelle attaque.

10. La Corée a toujours considéré les Mandchous, à l'origine de la dynastie des Qing, comme des barbares. Elle se soumettra toutefois officiellement au nouvel empereur après l'invasion qu'elle subit en 1636.

11. Nous empruntons ce terme à l'historien Nakao Hiroshi 中尾宏. Voir «Chōsen tsūshinshi to kinsei nihon sakoku shikan o koete» 朝鮮通信使と近世日本「鎖国」史観をこえて (Les Ambassades coréennes et le Japon de la période moderne - Au-delà de la vision de fermeture), in Ueda Masaaki 上田正昭 (dir.), Nihon to Chōsen no kankei shi - kodai kara gendai made 日本と朝鮮の 関係史古代から現代まで (Histoire des relations entre le Japon et la Corée - De l'Antiquité à nos jours), Agenda Purojekuto アジェンダ・プロジェクト, Tōkyō, 2004, 202 p., p. 73-95.

12. Ces termes impliquent une relation hiérarchique.

13. En japonais hitsudan 筆談 ou hitsugo 筆語, littéralement « conversation à l'aide du pinceau ». Aujourd'hui encore, un Japonais et un Chinois peuvent avoir recours à un hitsudan, mais en raison de l'évolution sémantique des sinogrammes, propre à chaque pays, il peut parfois y avoir des malentendus, comme le souligne Kazuhiko Watabe dans son article «Hitsudan: le dialogue par le pinceau ", dans le Courrier international, $\mathrm{n}^{\circ}$ 701, 8 avril 2004.

14. Il existe de nombreuses études sur les ambassades coréennes au Japon, et en particulier sur celle de 1711, car les relations entre Japonais et Coréens furent, à cette date, particulièrement difficiles en raison des réformes protocolaires apportées par Arai Hakuseki. La plupart des historiens ont cherché à mettre en lumière la pensée politique d'Arai Hakuseki, au sujet des relations avec les pays étrangers, et sa conception de la place du Japon en Asie, en analysant ces réformes : le changement de titre pour désigner le shōgun, le protocole pour l'accueil des envoyés coréens, etc. Sur la vie et l'œuvre d'Arai Hakuseki, nous renvoyons le lecteur aux ouvrages du spécialiste japonais Miyazaki Michio 宮崎道生, tous publiés chez Yoshikawa Kōbunkan 吉川弘文 館, à Tōkyō : Arai Hakuseki Joron 新井白石序論 (Introduction à Arai Hakuseki), (1954), 1976 (éd. revue et augmentée), 251 p. ; Arai Hakuseki 新井白石 (Arai Hakuseki), (1957), 1989 (éd. revue et augmentée), 326 p.; Arai Hakuseki no kenkyū 新井白石の研究 (Études sur Arai Hakuseki), (1958), 1987 (éd. revue et augmentée), 887 p. ; Arai Hakuseki no gendaiteki kōsatsu 新井白石の現代的考察 (Considérations de notre temps sur Arai Hakuseki), 1985, 292 p.; Arai Hakuseki dansōo 新井白石断 想 (Pensées sur Arai Hakuseki), 1987, 188 p. Nakamura Hidetaka 中村栄孝 a consacré, quant à lui, ses travaux sur les relations entre la Corée et le Japon. Dans le troisième et dernier volume de Nissen kankeishi no kenkyū 日鮮関係史の研究 (Études sur l'Histoire des relations nippocoréennes), Yoshikawa Kōbunkan, 1969, 698 p., p. 307-308, il aborde la question des réformes de 1711. De même, Ronald Toby traite de ces réformes en focalisant son attention sur l'attitude de la cour coréenne. Voir “Korean-Japanese diplomacy in 1711 Sukchong's Court and the Shogun's Title”, in Chōsen Gakuhō 朝鮮学報 (Bulletin d'Études coréennes), nº 74, 1975, pp. 1-26. Enfin, la thèse de Philippe Gravier, soutenue en 2004 à l'INALco, De l'empereur au shōgun : études sur les écrits d'un lettré confucianiste de l'époque d'Edo, Arai Hakuseki (1657-1735), est une bonne introduction à la pensée politique d'Arai Hakuseki. La question des réformes de 1711 y est brièvement abordée, p. 81-89.

15. Il s'agit du Tojō shishū 陶情詩集 (Recueil de poèmes de divertissement), compilé en 1681-1682.

16. Penseur néo-confucianiste comptant parmi ses disciples de grands lettrés de l'époque d'Edo : Arai Hakuseki, Amenomori Hōshū 雨森芳洲 (1668-1755), Muro Kyūsō 室鳩单 (1658-1734), mais aussi Sakakibara Kōshū 棉原筀洲 (1656-1706).

17. À la place du clan des Hayashi 林 (responsable des études au bakufu) qui, traditionnellement, s'occupait de cette question. 
18. Alors qu'en 1636 , on avait décidé du contraire.

19. Pour plus de détails sur les réformes d'Arai Hakuseki, voir Miyazaki Michio, Arai Hakuseki no kenkyū, op. cit., en particulier les chapitres deux et trois, ainsi que Nakamura Hidetaka, Nissen kankeishi no kenkyū, op. cit., p. 307-308.

20. Le Hakuseki Shisō 白石詩草 (Poèmes manuscrits de Hakuseki), compilé en 1711.

21. Yi Wŏn-sik 李元植, «Arai Hakuseki to Chōsen Tsūshinshi » 新井白石と朝鮮通信使 (Arai Hakuseki et les ambassades coréennes), in Miyazaki Michio, Arai Hakuseki no Gendaiteki Kōsatsu, op. cit., p. 140.

22. Cette pratique n'est pas limitée au Japon, comme le rappelle Daniel Bouchez dans son Histoire de la littérature coréenne des origines à 1919, Fayard, 2002, 425 p., p. 25-26. L'usage du chinois classique comme véhicule de la culture écrite en Asie peut évoquer le latin, à la différence près que le latin était une langue écrite et parlée.

23. On peut se faire une idée de l'ambiance d'une telle rencontre en regardant une estampe de Katsushika Hokusai 葛飾北斎 portant le titre de Yui 由井 (collection privée).

24. Collection des récits de voyage, en douze volumes, parue entre 1974 et 1981, publiée chez Minjok munhwa ch'ujin hoe 民族文化推進會. La version coréenne du Kōkan hitsudan se trouve dans le volume 9. Nous n'avons pas pu voir la version du Kyujanggak, mais selon Yi Il-jae, elle ne présente pas de différence. Nous n'avons pas pu lire la version imprimée du Tongsa Ilgi, mais la version en chinois classique et sa traduction en coréen proposée en ligne par l'Institut pour la traduction des Classiques coréens (http://db.itkc.or.kr/itkcdb/mainIndexIframe.jsp, consulté le 10 février 2010). On peut également consulter le manuscrit de la Bibliothèque nationale coréenne sur son site (http://www.nl.go.kr consulté le 10 février 2010).

25. Kokusho sōmokuroku 国書総目録 (Catalogue général des ouvrages nationaux, 8 volumes), vol. 2, p. 206.

26. Arai Hakuseki Zenshū 新井白石全集 (Æuvres complètes d'Arai Hakuseki), 6 volumes, Kokusho kankô-kai 国書刊行会, 1905-1907.

27. Du nom de l'ère japonaise Kansei 寛政, commençant en 1789.

28. Yi Il-jae 李一宰, «Kanggwan p'ildam e teahan ilgoch'al » 江關筆談에 대한 일고찰 (Réflexions sur le dialogue au fil du pinceau), dans la revue Asia Munhwa 아시아문화 (Culture d'Asie), $n^{\circ} 19$, Ch'unch' ŏn, Hallim taehakkyo Asia munhwa yŏnguso 翰林大學校아시아文化研究所, 2003, p. 163-205, p. 178. Il a montré ainsi que la version du Tongsa ilgi comporte 5515 caractères, dont 3134 caractères qui n'apparaissent que dans le texte japonais, tandis que la version kansei comporte 4280 caractères dont 1988 qui n'apparaissent pas dans le texte coréen.

29. Ibid., p. 199-201.

30. Ibid., p. 181.

31. Voir l'article de Shiomura Kō 塩村耕, «Amenomori Hōshū to Arai Hakuseki » 雨森芳洲と新 井白石 (Amenomori Hōshū et Arai Hakuseki), Bungaku 文学 (Littérature), mai-juin 2002, p. 3-13.

32. «Tsushima kuni ni aritsuru nama gakushōra» 対馬国二ありつるなま学匠等, in Oritaku shiba no ki 折りたく柴の記 (Récits autour d’un feu de bois, 1716), Iwanami Bunko, 1999, 476 p., p. 199.

33. Yi Wŏn Sik a présenté brièvement le Kōkan hitsudan dans «Arai Hakuseki to Chōsen Tsūshinshi », op. cit. Par la suite, il aborde de nouveau le texte dans son ouvrage Chosón t'ongsinsa 朝鮮通信使, Minŭnsa 民音社, 1991, 465 p., sans apporter malheureusement plus d'information. Chŏng Ŭng-su 鄭應洙 analyse, quant à lui, l'ethnocentrisme naissant des Coréens au XVIII siècle, en comparant le contenu de deux dialogues, celui des envoyés coréens avec Arai Hakuseki en 1711 , et celui des envoyés coréens avec des officiels Chinois. Voir « 18 saeki tongasia jubyon munhwagwon ŭi munhwajŏk jakak gwa junghwa sasang ŭi swoet'oe - Kanggwan Pil'dam gwa Hokjong Pil'dam ŭl jungsim ŭro » 18 세기 동아시아 주변 문화권의 문화적 자각과 중화사상의 쇠 퇴 —「강관필담」과「혹정필담」을 중심으로 (Déclin du concept de centralité chinoise et réveil culturel au sein de la sphère culturelle de l'Asie de l'Est au XVIII ${ }^{\mathrm{e}}$ siècle à travers le Kanggwan 
Pil'dam et le Hokjong Pil'dam), in Ilbon Munhwa Hakpo 日本文化學報 (Revue d'études sur la Culture du Japon), 韓國日本學會 Hanguk Ilbon Munhwa hakhoe (Société coréenne d'études de la culture du Japon), nº 3, 1997, p. 355-380.

34. Mais il donne une analyse ne se fondant que sur le texte de la version kansei, et à aucun moment, il ne fait référence à la version tōsa. Voir Arai Hakuseki joron, op. cit., p. 54-60.

35. Yi Il-jae, « Kanggwan p'ildam e teahan ilgoch'al », op. cit.

36. Yi Wŏn-sik, « Arai Hakuseki to Chōsen Tsūshinshi », in Arai Hakuseki no gendaiteki kōsatsu, op. cit., p. 135.

37. Miyasaki Michio distingue, quant à lui, les parties suivantes : les connaissances sur le monde extérieur, la question de la civilisation chinoise, la relation diplomatique entre les deux pays, le problème des interdits et la conservation des ouvrages sur les rites ; Arai Hakuskei joron, op. cit., p. 55.

38. Hakuseki se contente de répondre laconiquement : «c'est à cause de notre peuple [qui refuse de le montrer]. On n'y peut rien, c'est regrettable.»

39. Arai Hakuseki, Oritaku shiba no ki, op.cit., p. 71-72.

40. Courant de pensée qui s'inspire des œuvres du lettré chinois Zhu Xi 朱子 (1130-1200) et de son école.

41. Arai Hakuseki rédigea un texte intitulé Seiyō Kibun 西洋紀聞 (Compte rendu de discussions sur l'Occident), qu'il garda précieusement et qui ne fut publié qu'en 1882. Voir Jacqueline Pigeot, "Le prêtre et le lettré : un dialogue des cultures dans le Japon du XVIII siècle ", in Foi chrétienne et cultures, Axes. Recherches pour un Dialogue entre Christianisme et Religions, Paris, 1982, vol. 14, $\mathrm{n}^{\circ} 1-2$, p. 49-62. Voir également la thèse de Philippe Gravier mentionnée plus haut.

42. La dynastie Chosŏn, en particulier après l'invasion de la Corée par les guerriers d'Hideyoshi, préféra éviter les contacts avec les étrangers, car pour elle tout ce qui venait de l'étranger ne pouvait être synonyme que de calamité. Dans ce but, le gouvernement mit en place des gardecôtes pour repousser ou arrêter tout éventuel intrus, comme ce fut le cas pour les marins hollandais du navire marchant Sperwer, qui échoua sur les côtes de la province de Chŏlla, en 1653. Il faudra attendre le traité de Kanghwa, en 1876, pour que la Corée s'ouvre au monde extérieur. Cette politique d'isolement explique la pénurie d'information en Corée sur le monde, et surtout sur l'Occident.

43. À l'origine de la Mission catholique de Chine.

44. À partir de 1637, seuls les Hollandais et les Chinois étaient autorisés à résider au Japon dans un espace délimité à Nagasaki. Pour endiguer la fuite des métaux précieux, le nombre de navires autorisés à entrer à Nagasaki a été limité, en 1715, à 30 par an pour les navires chinois et seulement à deux pour les Hollandais. Mais il semble qu'au début du XviII siècle, le trafic ait été beaucoup plus important entre la Chine et le Japon, si l'on se réfère aux paroles d'Arai Hakuseki.

45. Hakuseki mentionne deux coiffes de l'époque des Yin, le shōho 章甫, dont Confucius aurait fait usage, et le fuku 料蒝 (chapeau brodé).

46. Nous reprenons les explications de l'historien Naitō Torajirō (Naitō Konan), telles que présentées par Yi Il-jae ou encore Miyasaki Michio, car nous n'avons pas pu lire directement son texte, «Hakuseki no ichi ibun ni tsuite» 白石の一遺聞について(Un aspect méconnu de Hakuseki), in Sentetsu no gakumon 先哲の学問 (Leçons des sages d'autrefois), Chikuma shobô 筑摩 書房, 1987, $254 \mathrm{p}$.

47. Miyasaki Michio, Arai Hakuseki joron, op. cit., p. 57.

48. Hakuseki avait sans doute lu des ouvrages coréens que l'on trouvait au Japon, tels que le Haedong chegukki 海東諸國記 (Au pays de l'Est de la mer, 1471), le Chingbirok 懲枈録 (Leçon pour l'avenir, 1695), le Kosa Ch'walyo 攻事撮要 (Notes sur les aspects importants du passé, 1554-1771), ou encore le Kyongguk Taejon 經國大典 (Code de lois pour diriger le pays, 1485), dans lesquels transparaît le mépris de leurs auteurs pour les armes, signe de barbarie à leurs yeux. 
49. Im Su-gan aurait fait cette requête lors de la première rencontre avec Hakuseki, à Kawasaki et, plus tard, lorsque les Coréens assistent, dans la résidence shogunale, à une réception faite en leur honneur, le troisième jour du onzième mois de l'année 1711.

50. Dans la version tōsa, on ne trouve pas ce long développement. Hakuseki se contente de répondre : « Notre pays accorde de l'importance à la chose militaire, mais vous le savez déjà ».

51. En effet, le onzième jour du même mois, les envoyés coréens ont la stupeur de voir, quand ils reçoivent la lettre officielle de réponse du shōgun, qu'il contient un caractère « eki » 懌 rappelant le nom de Chungjong 中宗 (1488-1544), ancien roi de Corée. Selon la tradition en vigueur en Asie du Nord-est, il était interdit d'utiliser les sinogrammes du nom personnel d'un roi ou d'un ancêtre défunt. Ils ne peuvent donc l'accepter en l'espèce, et demandent sur un ton ferme aux Japonais de corriger la lettre. Les Japonais, Hakuseki en tête, répliquent alors que c'est la Corée qui a commis en premier une faute en employant un caractère «mitsu » 光 qui rappelle celui de Tokugawa Iemitsu (1604-1651), et qu'il n'est pas question de changer les termes de la lettre en question. L'affaire sera résolue, non sans mal, sous la forme d'un compromis, le bakufu s'engageant à renvoyer aux envoyés une lettre de réponse, à condition que la cour coréenne renvoie à son tour une lettre officielle corrigée. Voir à ce sujet, Yamamoto Hirofumi 山本博文, Tsushimahan Edo karō 対馬藩江戸家老 (Le Fief de Tsushima et ses grands conseillers d'Edo), Tōkyō, Kōdansha gakujutsu bunko 講談社学術文庫, 2002, 328 p. Par ailleurs, Hakuseki relate l'incident dans son autobiographie, Oritaku shiba no ki, op. cit., p. 203, en ces termes : "Alors que nous avions remis la lettre de réponse [du shōgun aux Coréens], ils nous ont demandé de corriger l'insulte faite à la mémoire d'un de leur roi, à la septième génération, en raison de l'usage incorrect (imina), dans le texte, d'un caractère correspondant à son nom. Je leur ai répondu en ces termes: [On peut lire dans le Liking:] "Depuis les temps anciens, on respecte l'interdiction portant sur l'usage du nom d'un défunt pendant cinq générations." En outre, selon Confucius, c'est un devoir du fils envers son père, du sujet envers son souverain. C'est une façon d'entretenir les liens qui les unissent. Pourquoi dans la relation entre deux pays, les sujets d'un pays devraient-ils également respecter l'interdit concernant un souverain étranger ? Enfin, même s'il devait en être ainsi, on ne trouve dans le passé nul exemple de cet interdit qui porterait sur sept générations. Ne dit-on pas en outre "abstiens-toi de faire ce tu ne veux pas qu'on te fasse" ? Or dans leur lettre même, on constate qu'ils ont enfreint l'interdit, envers Tokugawa Iemitsu. Ils ont beau jeu de nous rappeler l'interdit portant sur sept générations, quand eux-mêmes ne le respectent pas. Leurs réclamations étaient inacceptables (...)». De retour dans leur pays, les envoyés coréens seront jugés et châtiés, la cour coréenne estimant qu'ils avaient failli à leur devoir en manquant de fermeté à l'égard des Japonais sur un sujet aussi sensible que celui des noms.

52. Il y avait, parmi les membres du cortège coréen, des interprètes qui avaient passé un concours pour obtenir ce poste. Du côté japonais, des interprètes étaient formés sur l'île de Tsushima puis envoyés en stage dans le comptoir japonais (wakan 倭館), à Pusan. Soucieux de former de bons interprètes japonais, Amenomori Hōshū écrivit le premier manuel de coréen au tout début XVIII ${ }^{\mathrm{e}}$ siècle, le Kōrin suchi 交隣須知 (Des Connaissances de base pour les relations diplomatiques). Celui-ci resta en usage jusqu'à la fin la période Meiji.

53. Les descendants de Hayashi Razan (1583-1657).

54. Shin Yu-han 申維翰 (1681- ?) a écrit le Haeyurok 海遊録 (Journal d'un voyage maritime) au cours de son voyage au Japon en 1719 (An 4 de l'ère Kyōhō 享保) dans le cadre d'une ambassade coréenne. À propos des remarques de Shin Yu-han, voir Kamigaitō Ken.ichi 上坦外憲一, Amenomori Hōshū 雨森芳洲 (Amenomori Hōshū), Tōkyō, Chūkō Shinsho, 2005, 256 p., p. 162.

55. Nakao Hiroshi écrit à ce sujet que c'est la première fois, à l'époque d'Edo, que des écrits japonais sont transmis à l'étranger. Voir Chōsen tsushinshi 朝鮮通信使 (Les Ambassades coréennes), Tōkyō, Iwanami Shinsho 岩波新書, 2007, 208 p., p. 155-156. 
56. D'ailleurs, Shin Yu-han raconte, au début de son récit, qu'une fois arrivé à Séoul, il a rencontré un lettré qui lui a remis un exemplaire d'une œuvre poétique de Hakuseki, en faisant l'éloge de ce dernier.

57. «Chōsen heishi gogi » 朝鮮聘使後議 (Réflexions après la venue des envoyés coréens, 1712), in Arai Hakuseki zenshū, op. cit., vol. 4.

58. Inoue Atsushi 井上厚史 constate ainsi une certaine continuité entre les écrits de Hakuseki et l'émergence d'une pensée nationale via les écrits d'auteurs tels que Motoori Norinaga 本居宣長 (1730-1801). Voir son article, «Arai Hakuseki no chōsen kan » 新井白石の朝鮮観 (Arai Hakuseki et sa perception de la Corée), Kan 環, nº 23, Fujiwara Shoten 藤原書店, Tōkyō, 2005, p. 114-125.

\section{RÉSUMÉS}

Durant l'époque d'Edo, plusieurs ambassades coréennes se rendent dans la capitale shogunale. De ces importants échanges culturels témoignent les « conversations au fil du pinceau » (hitsudan).

During the Edo period, several Korean ambassadors visited Japan. The "brush conversations" (hitsudan) are a testimony of the two countries' cultural exchanges.

\section{INDEX}

Index géographique : Corée, Tsushima キーワード : Arai Hakuseki 新井白石 (1657-1725), Tokugawa bakufu 徳川幕府 (1603-1867), hitsudan 筆談, Kōkan hitsudan 江關筆談, gaikō 外交, Kankoku 韓国, Tsushima 対馬, Edo jidai 江 戸時代 (1603-1867), rekishi 歴史

Index chronologique : Edo (1603-1867)

Keywords : Arai Hakuseki (1657-1725), Tokugawa shogunate (1603-1867), hitsudan, Kōkan hitsudan, Diplomacy, Korea, Tsushima, Edo Period (1603-1867), History

Mots-clés : Arai Hakuseki (1657-1725), shogunat Tokugawa (1603-1867), hitsudan, Kōkan hitsudan, diplomatie

Thèmes : histoire 